\title{
Consumo de Psicofármacos em uma Região Administrativa do Rio de Janeiro: A llha do Governador
}

\section{Consumption of Psychotropic Drugs in an Administrative Region of the City of Rio de Janeiro: Illha do Govemador}

\author{
Liz Maria de Almeida ${ }^{1}$ \\ Evandro da S. F. Coutinho \\ Vera Lúcia E. Pepe ${ }^{3}$
}

ALMEIDA, L. M.; COUTINHO, E. S. F. \& PEPE, V. L. E. Consumption of Psychotropic Drugs in an Administrative Region of the City of Rio de Janeiro: Ilha do Governador. Cad. Saúde Públ., Rio de Janeiro, 10 (1): 05-16, Jan/Mar, 1994.

In 1988, a cross-sectional survey was conducted to estimate the prevalence of alcoholism and alcohol consumption in the 20th Administrative Region of the city of Rio de Janeiro. The interview protocol included questions about consumption of psychotropics, coffee, and cigarettes. This paper presents data from psychotropic consumption in the month. The sample of the population older than 13 years old included 1,459 subjects. Overall consumption of psychotropic drugs was $5.2 \%$. Rate of psychotropic use was higher for females (6.7\%) than for males (3.1\%). Users were predominantly women, elderly, (the highest rate was for those between 60-69 years), divorced or widows, and with lower family income. The association of each socio-demographic variable was evaluated by the odds ratio adjusted by logistic regression. Anxiolytics (benzodiazepines) predominated (85.23\%) among the reported psychotropics, followed by antiepileptics (5.68\%) and hypnotics and sedatives (4.54\%). Physicians not specializing in neurology or psychiatry lead prescriptions (65.8\%). $80.26 \%$ of the drugs were obtained in pharmacies, while $13.16 \%$ were obtained in governmental institutions, most of them by controlled prescriptions. The results are discussed and new lines of research are suggested.

Key words: Psychotropics; Anxiolytics; Epidemiology; Prevalence; Consumption

\section{INTRODUÇÃO}

No ano de 1988 foi realizado um inquérito epidemiológico na XX Região Administrativa (RA) do município do Rio de Janeiro, que com-

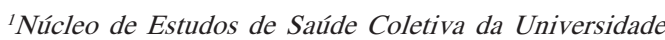
Federal do Rio de Janeiro. Av. Brigadeiro Trompowski $s / n^{o}$, Prédio do Hospital Universitário Clementino Fraga Filho, $5^{\circ}$ andar, Ala Sul, Ilha do Fundão, RJ, 21949-900, Brasil.

${ }^{2}$ Departamento de Epidemiologia e Métodos Quantitativos em Saúde da Escola Nacional de Saúde Pública. Rua Leopoldo Bulhões 1.480, $8^{\circ}$ andar, Rio de Janeiro, RJ, 21041-210, Brasil.

${ }^{3}$ Departamento de Administração e Planejamento em Saúde da Escola Nacional de Saúde Pública. Rua Leopoldo Bulhões 1.480, $7^{\circ}$ andar, Rio de Janeiro, RJ, 21041-210, Brasil. preende a Ilha do Governador, a Ilha do Fundão e mais 62 ilhas e ilhotas. O objetivo principal do inquérito era estimar a prevalência de alcoolismo crônico na região. O consumo de psicofármacos, bem como de cigarros e café, foi inserido no estudo com uma dupla finalidade: correlacionar-se com o consumo de álcool e, por outro lado, minimizar o impacto das questões referentes ao alcoolismo crônico dentro do questionário. Este trabalho apresenta os resultados referentes ao consumo de psicofármacos.

A psicofarmacologia teve início com os trabalhos de Emil Kraepelin, mas as descobertas que revolucionaram a prática clínica somente ocorreram após a Segunda Guerra Mundial. O meprobamato, os sais de lítio, a clorpromazina e os diversos neurolépticos que a seguiram, os 
inibidores da monoaminoxidase (IMAO), os antidepressivos tricíclicos, o clordiazepóxido e os derivados benzodiazepínios diminuíram significativamente o número de internações psiquiátricas e o tempo de permanência dos pacientes psiquiátricos nos hospitais. Além disso, permitiram que pacientes com história de diversas internações regressassem a seus lares e pudessem continuar seus tratamentos em ambulatórios. Para os profissionais de saúde, os avanços da psicofarmacologia reaproximaram a especialidade da prática clínica geral, trazendo não apenas novas perspectivas terapêuticas, mas a responsabilidade de se aprimorar os diagnósticos e as ações psicofarmacológicas dos medicamentos e de reduzir os riscos envolvidos na sua utilização (Gentil-Filho, 1992; Rocha, 1992; Pinder, 1992).

Interferindo neste espaço terapêutico, as questões que envolvem a política de medicamentos, tais como aquisição, distribuição, legislação, controle de qualidade, relação com as indústrias farmacêuticas internacionais, acesso e eqüidade, vêm assumindo uma dimensão dia a dia maior (OMS, 1990; CEME/ MS, 1987; Lucchesi, 1991; Tancredi, 1986; Medici et al., 1991).

A Organização Mundial da Saúde (OMS, 1990) refere que, em $1985,25 \%$ da população mundial consumiam mais de três quartos dos medicamentos produzidos no mundo. Neste mesmo ano, o Brasil foi classificado entre os 46 países do Grupo C - países de cobertura medicamentosa elevada. Uma série de requisitos foram preenchidos para que o país se inserisse neste nível: o acesso aos medicamentos essenciais (60-90\% da população); a presença de uma lista de medicamentos essenciais; a existência de uma política farmacêutica nacional; a existência de uma legislação farmacêutica; a existência de um laboratório oficial para controle de qualidade; a existência de um sistema de distribuição dos medicamentos essenciais; a existência de uma produção de substâncias químicas; estar situado entre uma determinada faixa do PIB per capita, etc.

Em contraposição aos dados da OMS, a Central de Medicamentos do Ministério da Saúde (CEME/MS, 1987) publicou um documento sobre medicamentos essenciais onde afirma que somente $48 \%$ da população brasileira têm acesso aos remédios. Medici et al. (1991), em um trabalho sobre a política de medicamentos no Brasil, divulgaram dados do Plano Nacional de Amostragem Domiciliar (PNAD), de 1986, onde observamos que apenas $14,6 \%$ das pessoas que procuraram atendimento em serviços de saúde e receberam uma prescrição conseguiram obter os medicamentos na rede pública; $11,3 \%$ conseguiram parte das medicações; e 74,1\% não conseguiram nenhum medicamento por esta via.

A despeito disso, em 1989 o Brasil ocupava o $8^{\circ}$ lugar entre os 10 principais mercados farmacêuticos do mundo capitalista (Rozenfeld, 1989). No entanto, o consumo anual per capita, de cerca de 17 dólares, aproxima o país daqueles cuja distribuição medicamentosa se dá de tal forma que apenas $23 \%$ da população consomem $60 \%$ da produção (Bermudez, 1992). Em termos de América Latina, o consumo brasileiro é semelhante ao da Argentina, cuja população é cinco vezes menor (Medici et al., 1991).

Os medicamentos destinados ao tratamento das doenças mentais ocupavam, em 1985, o $9^{\circ}$ lugar entre os mais vendidos mundialmente, representando 6,9\% das vendas. Só no Brasil, em 1986 consumiram-se 500 milhões de doses diárias de tranqüilizantes, o que, segundo a Organização Mundial da Saúde (OMS) representava uma quantidade três vezes superior às suas necessidades (OMS, 1990).

Dentre os psicofármacos, os benzodiazepínicos encontram-se entre os mais consumidos, tanto em países desenvolvidos quanto em países em desenvolvimento (Laporte et al., 1983; Tancredi, 1986; Guereje \& Obikoya, 1991).

Os estudos populacionais sobre consumo de psicofármacos são relativamente escassos em nosso país (Tancredi, 1979; Mari et al., 1993), o que nos obriga, muitas vezes, a lançar mão de estatísticas internacionais para o planejamento de ações de saúde em nosso área de atuação. Além disso, o panorama do consumo de psicofármacos de uma população pode fornecer outras informações indiretas, como, por exemplo, a prevalência de morbidade (Woods et al., 1988) ou o impacto dos programas de controle no consumo dos medicamentos. 


\section{MATERIAL E MÉTODO}

Para estimarmos a prevalência do consumo de psicofármacos foram reanalisados os dados de um estudo de delineamento transversal (inquérito epidemiológico) na população de maiores de 13 anos da XX Região Administrativa (Ilha do Governador) do município do Rio de Janeiro. Em 1988, ano do estudo, esta população estava estimada em cerca de 250.000 habitantes. A população da Ilha do Governador está distribuída por 15 bairros e 13 favelas, não se apresentando homogênea quanto ao nível sócioeconômico (Fibge, 1983). Como psicofármacos consideramos todas as preparações que faziam parte da lista de medicamentos controlados, segundo as Portarias DIMED 27 e 28, de 1986.

\section{Amostras}

\section{Delineamento}

Utilizou-se um delineamento amostral em três estágios, por conglomerados. No primeiro estágio sortearam-se 59 setores censitários de um total de 183 , sorteio este com probabilidade proporcional ao tamanho de cada conglomerado. Foram excluídos os setores coletivos (hospitais, quartéis, escolas, igreja). Em cada conglomerado foi feita uma visita para se atualizar os mapas de ruas (Fibge, 1983) e registrar os endereços de todos os domicílios. Em seguida, foram sorteados 30 domicílios em cada setor dos quais selecionou-se um indivíduo aleatoriamente.

\section{Tamanho}

O interesse primário do estudo era a prevalência de alcoolismo crônico, com um erro amostral de $2 \%$, o que determinou um tamanho amostral de 1.800 indivíduos com mais de 13 anos. Os parâmetros para esta estimativa encontram-se em outro trabalho (Almeida \& Coutinho, 1993).

\section{Coleta de Dados}

\section{Instrumento}

Para este fim, utilizou-se um questionário com 52 perguntas fechadas, que versavam sobre os seguintes dados: variáveis demográficas e sócio-econômicas; uso de álcool, cigarro, café e psicofármacos; o questionário CAGE para alcoolismo crônico (Masur \& Monteiro, 1983), hábitos alimentares, ritmo de sono e lazer. Em relação aos psicofármacos, o questionário continha perguntas sobre o tipo de psicofármaco, o agente da indicação, o local de aquisição, o número de dias em que foram utilizados na última semana e nos últimos 30 dias, o controle da aquisição, mediante a apresentação de receituário especial obrigatório, e as medicações que não foram encontradas.

\section{Entrevistas}

Para se mapear os setores censitários e aplicar os questionários, 18 estudantes da área biomédica da Universidade Federal do Rio de Janeiro foram treinados pela equipe de profissionais, composta por uma psiquiatra e dois psicólogos. A atualização dos mapas durou 3 meses e a coleta de dados, incluindo até três visitas a cada domicílio, 4 meses.

\section{Análise dos Dados}

Inicialmente foram feitas tabulações para se estimar a prevalência instantânea (últimos 30 dias) geral do consumo de psicofármacos, bem como a prevalência segundo as variáveis sóciodemográficas sexo, idade, situação conjugal, escolaridade e renda.

A associação das variáveis sócio-demográficas com o consumo de psicofármacos foi avaliada, inicialmente, através de olds ratios (ORs) obtidos através de análise simples. Os intervalos de confiança para $95 \%$ foram estimados através do método de aproximação de Cornfield (Kahn \& Sempos, 1989). Em seguida, foi feita uma primeira avaliação da presença de fatores potencialmente confounding e de interações para as associações de interesse, através da técnica estratificada. Estimaram-se, então, o odds ratio de Mantel-Haenszel (ORmh), seus respectivos intervalos de confiança e o p-valor. Por fim, procedeu-se à análise multivariada, através de regressão logística (Hosmer Jr \& Lemeshow, 1989). 
As cinco variáveis que entraram no modelo foram assim codificadas, com base nas análises preliminares: a) gênero (masculino, feminino); b) idade (14-34 anos, 35-54 anos, mais de 54 anos); c) escolaridade (baixa - até $1^{\circ}$ grau completo -, alta $-2^{\circ}$ grau completo ou mais); d) situação conjugal (solteiro, casado, separado/viúvo); e) renda (até três salários mínimos, mais de três salários mínimos).

Utilizou-se o sistema de classificação recomendado pelo WHO Drug Utilization Research Group, denominado Anatomical-TherapeuticChemical Classification System (ATC). Esta classificação é a sugerida para os estudos de utilização de medicamentos e vem sendo em - pregada em vários outros países (WHO/Nordiska Lakemedelsnamnden, 1993).

\section{RESULTADOS}

Na Tabela 1 observamos a composição da amostra segundo algumas variáveis sócio-demográficas. Houve uma predominância do gênero feminino $(59,7 \%)$, de menores de 40 anos $(59,7 \%)$ e de casados $(60,2 \%)$. Quatrocentos e sessenta e um entrevistados $(31,6 \%)$ não completaram o primeiro grau e $31,9 \%$ referiram uma renda familiar de, no máximo, três salários mínimos.

TABELA 1. Prevalência de Consumo de Psicofármacos nos Últimos 30 Dias, segundo Variáveis Sócio-Demográficas e Níveis de Significância Estatística

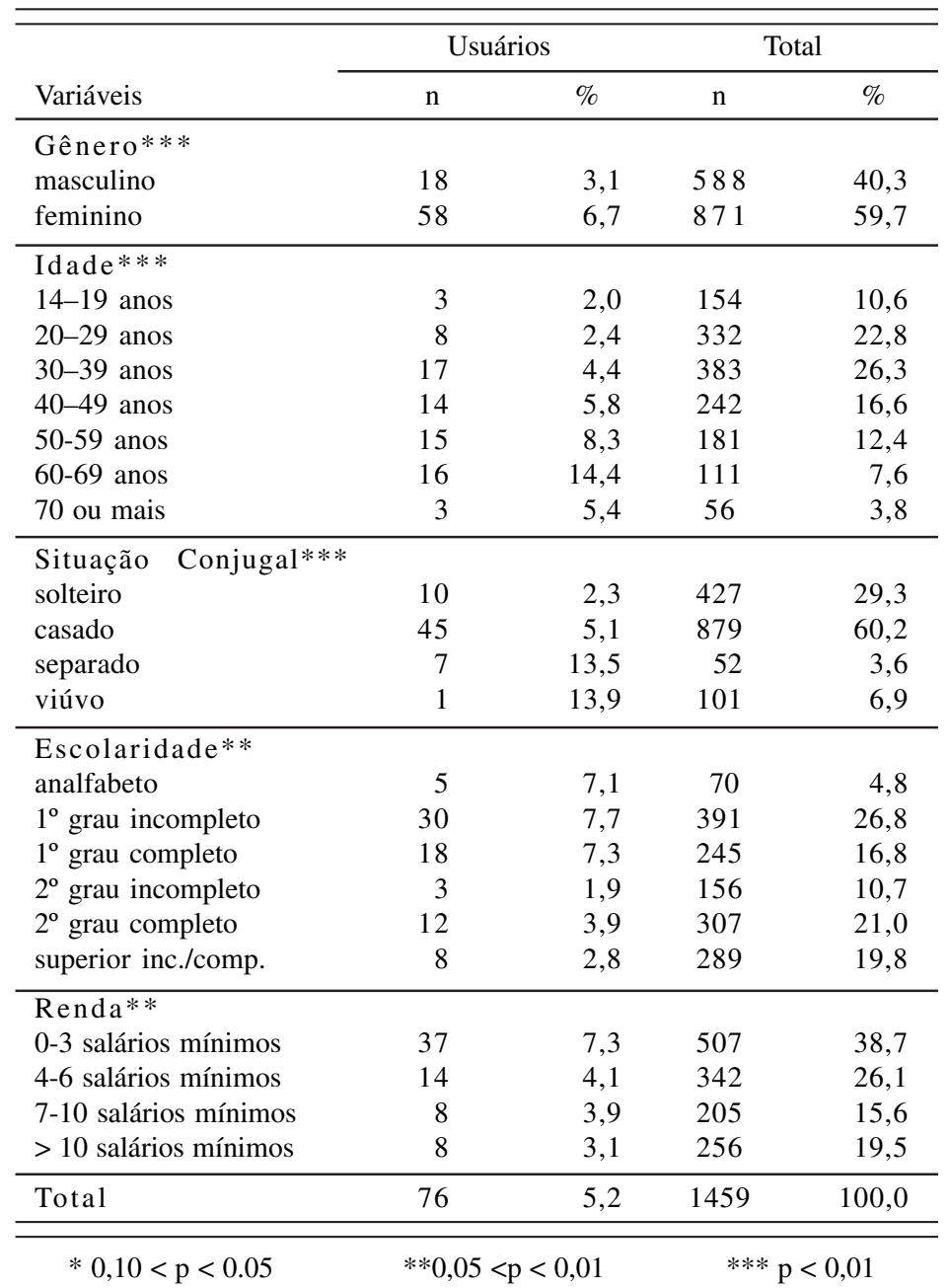


O uso de algum tipo de medicação nos últimos 30 dias, independente do tipo ou origem (alopática, homeopática, ervas, etc), foi referido por $15,4 \%$ da população, sendo que $33,8 \%$ deste grupo fizeram uso de psicofármacos, associados ou não a outros medicamentos.

O uso de psicofármacos nos últimos 30 dias foi referido por $5,2 \%$ dos indivíduos, sendo $6,7 \%$ do gênero feminino e $3,1 \%$ do gênero masculino (Tabela 1). Entre as mulheres, as donas de casa apresentaram um consumo de $7,7 \%$, enquanto a prevalência nas que trabalhavam fora de casa foi de 5,7\% ( $\mathrm{p}=0,30)$. A proporção de usuários nos últimos 30 dias também foi mais elevada entre os separados e viúvos, e menor entre os solteiros. Observou-se, ainda, uma redução no uso de psicofármacos com o aumento da renda e da escolaridade, sobretudo a partir do $2^{\circ}$ grau. Os dados mostram que a utilização destas substâncias aumentou com a idade, mas, enquanto no grupo das mulheres observou-se um aumento gradativo, a partir dos 20 anos, no grupo dos homens o aumento do consumo ocorreu mais tardiamente, a partir dos 50 anos. No entanto, para ambos os gêneros, o maior nível de consumo ocorreu na faixa etária de 60 a 69 anos, voltando a decrescer a partir dos 70 anos (Figura 1).
$\mathrm{Na}$ Tabela 2 encontram-se os odds ratios (ORs) brutos (não-ajustados) e aqueles obtidos para cada variável, com as demais controladas simultaneamente através de regressão logística. Pode-se observar que, para as mulheres, o OR pouco se alterou com o controle dos outros fatores. Os indivíduos mais velhos, os separados e viúvos, assim como aqueles de menores níveis de renda e escolaridade, também mantiveram-se associados positivamente com o uso de psicofármacos, tendo ocorrido um certo grau de enfraquecimento das associações.

$\mathrm{Na}$ Tabela 3 apresentam-se as proporções de cada tipo de psicofármaco entre as 88 substâncias relatadas. Observamos que os ansiolíticos foram os mais consumidos $(85,23 \%)$, seguidos pelos antiepilépticos $(5,68 \%)$. Dentre os ansiolíticos benzodiazepínicos referidos, destacou-se o diazepam como substância única ou associada $(65,4 \%)$. Outros derivados encontrados foram o bromazepam $(21,4 \%)$, o lorazepam $(5,3 \%)$, o clordiazepóxido (4,0\%), o alprazolam, o clobazam e o clorazepato dipotássico $(1,3 \%)$.

Esses 88 medicamentos eram consumidos por 76 pessoas, o que significa que havia 10 pessoas utilizando mais de uma substância (duas delas em uso de três substâncias concomitantemente). Porém, só havia uma pessoa em uso

FIGURA 1. Consumo de Psicofármacos nos Últimos 30 dias segundo o Grupo Etário, por Gênero

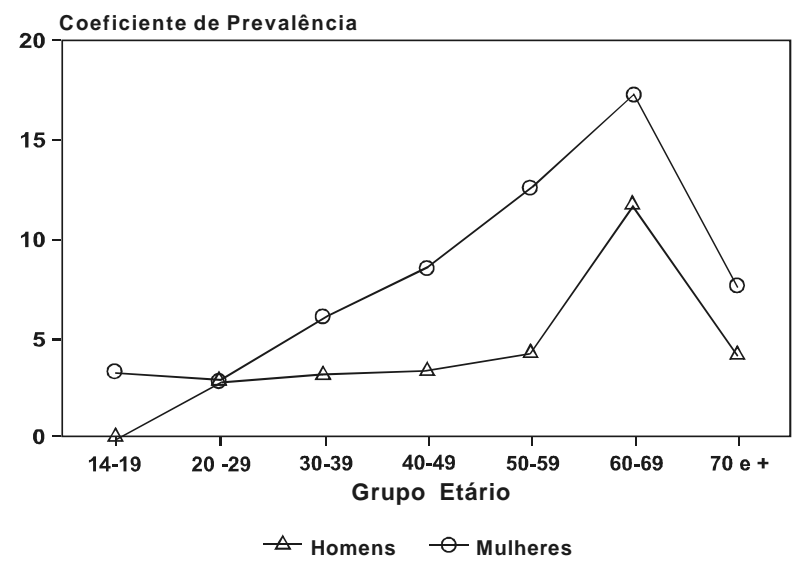

Fonte: XX RA do Rio de Janeiro, 1988. 
TABELA 2. Associação entre Consumo de Psicofármacos nos Últimos 30 Dias e Variáveis Sócio-Demográficas. Odds ratio Bruto $(\mathrm{ORb})$ e Ajustado por Regressão Logística (ORa), Intervalos de Confiança a 95\% (IC) e p-valor

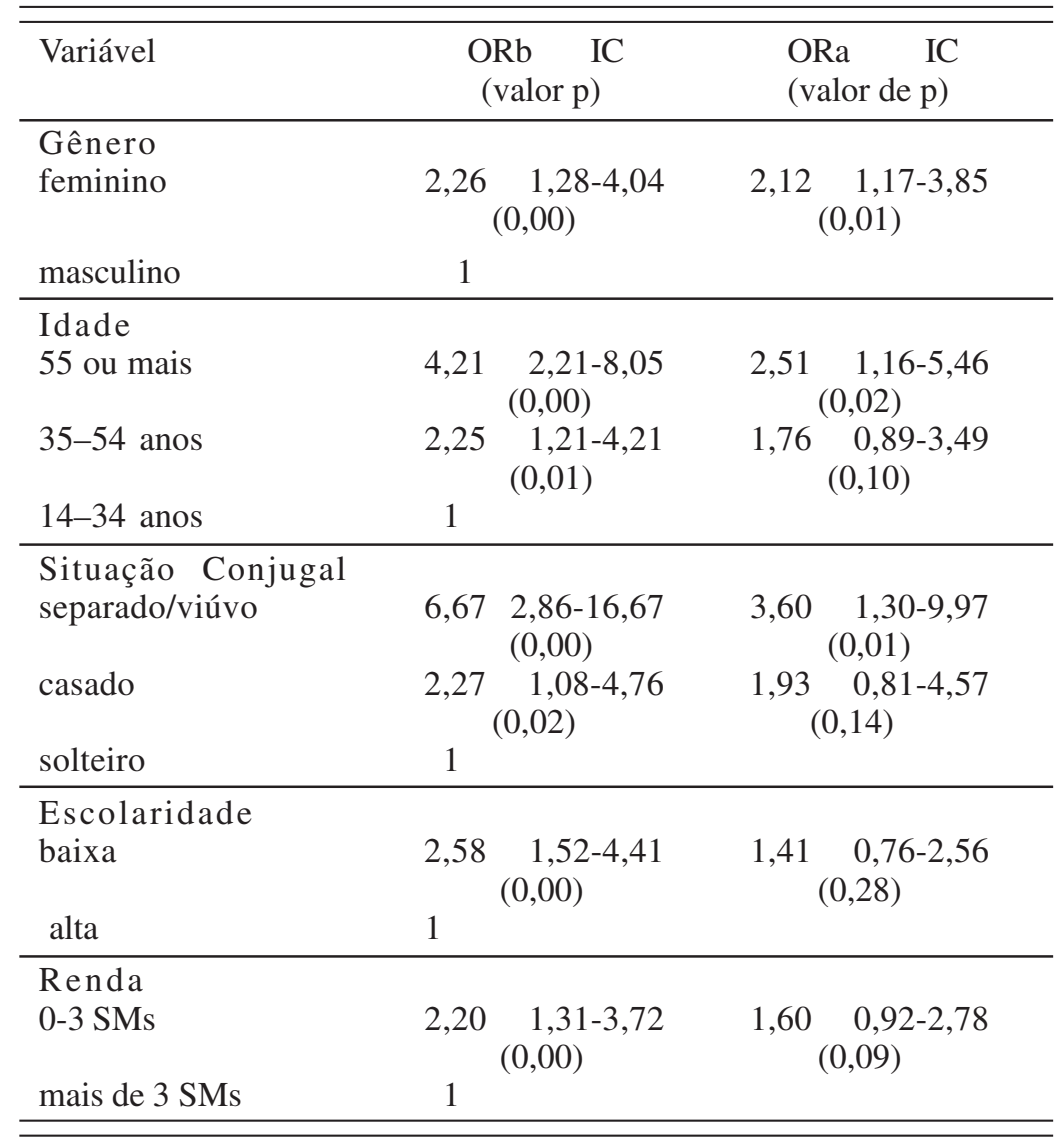

Obs.: O valor na coluna das RPC indica que a categoria foi tomada como referência.

isolado de outra substância que não um benzodiazepínico. Tivemos, portanto, 75 pessoas $(98,7 \%)$ em uso de benzodiazepínicos, como monoterapia ou associado a outras substâncias psicoativas, dentre as 76 que referiram uso de psicofármacos. A taxa de prevalência do uso de benzodiazepínicos foi de $5,14 \%$.

Em relação ao agente da indicação destes medicamentos, observamos que o médico de clínica geral, ou especializado em áreas que não neurologia e psiquiatria, foi responsável, isoladamente, por $65,8 \%$ das prescrições. Os especialistas (neurologista e psiquiatra) foram responsáveis por $23,7 \%$ das indicações. Ambos (clínico + especialista) prescreveram para o mesmo paciente em $6,6 \%$ dos casos, e apenas $3,9 \%$ obtiveram as medicações por outros meios.

Quanto à aquisição de psicofármacos, observamos que $80,26 \%$ os compraram em farmácias e/ou drogarias do comércio varejista; $13,16 \%$ conseguiram as medicações em farmácias de hospitais e postos públicos de saúde; e o restante $(6,54 \%)$ ou conseguiu na empresa onde trabalhava ou de alguma outra forma.

Houve referência à aquisição de quatro benzodiazepínicos $(5,2 \%)$ sem a apresentação obrigatória do receituário especial. Por outro lado, medicações que dispensam este documento só foram obtidas mediante a sua apresen- 
tação, como alguns anticonvulsivantes e o antidepressivo.

Quanto ao padrão de uso destas substâncias, observamos que 50\% dos usuários de benzodiazepínicos tiveram um consumo diário na última semana e 35,5\% fizeram uso diário nos últimos 30 dias. Os anticonvulsivantes foram tomados regularmente.

Não houve diferenças importantes quanto ao uso de psicofármacos nos últimos 30 (trinta) dias entre indivíduos que fumavam mais de 10 (dez) cigarros por dia $(5,9 \% \times 5,1 \%$; $=$ $0,62)$. As diferenças entre os consumidores de mais de 10 (dez) cafezinhos por dia e os que consumiram menos $(7,2 \%$ X 5,0\%; $p=0,22)$, ou entre aqueles que consumiam mais de 10 (dez) cafezinhos por dia e fumavam mais de 10 (dez) cigarros e os demais $(7,0 \%$ X 5,1\%; p = $0,29)$, não demonstraram significância estatística elevada.

TABELA 3. Psicofármacos Consumidos nos Últimos 30 Dias na XX RA do Rio de Janeiro - $1988(\mathrm{n}=88)$

\begin{tabular}{|c|c|c|c|}
\hline Tipo de Produto (segundo ATC*) & $\mathrm{N}$ & $\%$ & $\% /$ Total \\
\hline I.N03 - Antiepiléticos & & & 5,68 \\
\hline N03AA02 - Fenobarbital & 2 & 40,0 & \\
\hline N03AF01 - Carbamazepina & 3 & 60,0 & \\
\hline Sub-total & 5 & 100,0 & \\
\hline \multicolumn{4}{|l|}{ II.N05 - Psicolépticos } \\
\hline N05B - Ansiolíticos & & & 85,23 \\
\hline N05BA01 - Diazepam & 49 & 65,4 & \\
\hline N05BA02 - Clordiazepóxido & 3 & 4,0 & \\
\hline N05BA05 - Clorazepato dipotássio & 1 & 1,3 & \\
\hline N05BA06 - Lorazepam & 4 & 5,3 & \\
\hline N05BA08 - Bromazepam & 16 & 21,4 & \\
\hline N05BA09 - Clobazam & 1 & 1,3 & \\
\hline N05BA12 - Alprazolam & 1 & 1,3 & \\
\hline Sub-total & 75 & 100,0 & \\
\hline N05C - Hipnóticos e Sedativos & & & 4,54 \\
\hline N05CB02 - Fenobarbital (combinado) & 2 & 50,0 & \\
\hline N05CD01 - Flurazepam & 1 & 25,0 & \\
\hline NO5CD03 - Flunitrazepam & 1 & 25,0 & \\
\hline \multicolumn{4}{|l|}{ III.N06 - Psicoanalépticos } \\
\hline N06A - Antidepressivos & & & 1,14 \\
\hline N06AA02 - Imipramina & 1 & 100,0 & \\
\hline N06aC - Psicoanaléptico + Psicoléptico & & & 2,27 \\
\hline N06CA01 - Amitript. + Clordiazepóxido & 1 & 50,0 & \\
\hline N06CA** - Sulpiride + Bromazepam & 1 & 50,0 & \\
\hline Sub-total & 2 & 100,0 & \\
\hline \multicolumn{4}{|l|}{ IV - Outros: C03 - Diuréticos } \\
\hline C03AH - Tiazida em comb. c/ Psicoléptico & & & 1,14 \\
\hline C03AH** - Bendroflumetiaz. + Flufenazina & 1 & 100,0 & \\
\hline
\end{tabular}

* Guidelines for ATC Classification (WHO/Nordiska \& lakemedelsnamnden, 1993).

** Código específico não localizado.

Obs.: O sulpiride é um neuroléptico, mas, em doses baixas, é classificado como antidepressivo. 


\section{DISCUSSÃO}

Os resultados obtidos neste inquérito devem ser avaliados com cuidado, uma vez que o desenho da amostra foi projetado para o estudo do alcoolismo crônico. No entanto, acreditamos que este fato não compromete de modo importante os achados, na medida em que as prevalências de alcoolismo utilizadas para o cálculo amostral são praticamente as mesmas que aquelas do consumo de psicofármacos.

Outro ponto a ser mencionado é que a maior parte dos estudos populacionais sobre consumo de psicofármacos trabalha com a taxa de prevalência anual, o que dificulta comparações com este estudo, que registra a taxa de prevalência mensal deste consumo. Entretanto, julgamos que a avaliação do uso mensal dê origem a informações mais fidedignas, por estar a mesma menos sujeita ao viés de memória.

Neste inquérito, encontramos uma taxa de prevalência do uso de psicofármacos, nos últimos 30 dias, da ordem de 5,2\%. No Brasil, Tancredi (1979) encontrou uma taxa de prevalência mensal do uso de psicofármacos de $8,48 \%$ para a população da cidade de São Paulo. Uma importante diferença pode justificar a taxa mais baixa encontrada no nosso estudo: a introdução das Portarias DIMED 27 e 28, de 1986, pelo Ministério da Saúde, as quais regulamentaram a aquisição dos psicofármacos mediante a apresentação de um receituário médico especial. Estudos levados a cabo em outros países também demonstraram uma queda importante de consumo após a introdução de programas de controle de utilização de psicofármacos (Appelbaum, 1992; Shader et al., 1991).

Por outro lado, observamos que $98,7 \%$ dos usuários fizeram uso de pelo menos um benzodiazepínico nos últimos 30 dias, com ou sem associação com outros psicofármacos. Neste caso, foram encontrados resultados próximos aos obtidos no inquérito nacional de prevalência do uso de benzodiazepínicos no Canadá (1989), onde encontrou-se uma taxa de prevalência mensal de $5,7 \%$. Os levantamentos de prevalência mensal do uso de benzodiazepínicos, em diversos países, registram taxas entre 5 e $8 \%$ (Woods et al., 1992).

Parece não haver discordância, na literatura, quanto à predominância do consumo de psico- fármacos - sobretudo ansiolíticos - pelas mulheres, bem como quanto ao aumento deste consumo com a idade (Tancredi, 1979; Mari et al., 1992; Mellinger et al., 1984; North et al., 1992; Cafferata \& Meyers, 1990). Diversos autores referem que as mulheres são mais perceptivas em relação à sintomatologia das doenças, procuram precocemente ajuda e são menos resistentes ao uso de medicamentos prescritos do que os homens, o que poderia conduzir a uma chance maior de consumo de medicamentos, entre os quais os psicofármacos (Cafferata \& Meyers, 1990; Mellinger et al., 1984; Boltanski, 1989). Por outro lado, entre as mulheres é maior a freqüência de distúrbios psíquicos, condições circulatórias e doenças músculo-esqueléticas, para as quais é comum a prescrição de psicofármacos, sobretudo benzodiazepínicos. Estudos específicos com os usuários de benzodiazepínicos confirmam a maior frequiência não apenas de distúrbios psíquicos, mas também de problemas clínicos crônicos, como doenças cardiovasculares e artrites, tendinites e bursites, entre as mulheres (Mellinger et al., 1984). Há também uma maior relação de gênero feminino e idades mais avançadas com os distúrbios severos do sono (Mellinger et al., 1985).

Outro fator que poderia estar correlacionado com o gênero feminino é o maior comparecimento das mulheres às unidades de saúde, sobretudo em idade fértil (Madureira et al., 1989).

O aumento no consumo de psicofármacos com a idade parece estar ligado com a maior possibilidade de aparecimento de diversos problemas de saúde, entre os quais aqueles em que se utilizam os psicofármacos como terapia principal ou como coadjuvantes. Isto, porém, não explica o motivo pelo qual, a partir dos 70 anos, o consumo de psicofármacos diminui (Figura 1). Esta queda foi também observada por Tancredi (1979), no Brasil, e em estudos regionais realizados na Itália (Woods et al., 1992). Entretanto, pode-se considerar a hipótese de que os indivíduos que ultrapassam a faixa dos 70 anos seriam física e psiquicamente mais saudáveis. Tal achado seria, segundo esta hipótese, o resultado de um processo seletivo entre as pessoas que alcançaram idades acima da expectativa de vida do país. Estudos especí- 
ficos com este grupo populacional merecem ser realizados para esclarecer os motivos da redução no consumo de medicamentos em faixas etárias acima de 69 anos. Outra possível explicação seria o pequeno número de indivíduos nesta faixa etária, o que torna os dados muito instáveis.

Em relação à situação conjugal, encontramos uma taxa de prevalência maior entre aqueles que já tiveram um cônjuge e o perderam, por motivo de morte ou separação. Os solteiros apresentaram os menores índices de consumo, enquanto os casados situaram-se em uma faixa intermediária. Estes dados também estão presentes na literatura (Cafferata \& Meyers, 1990) e parecem sugerir que a perda de um companheiro constitui-se um fator de risco para o uso de psicofármacos. É interessante observar que a literatura epidemiológica costuma identificar uma prevalência menor de transtornos mentais entre indivíduos casados. Porém, um estudo realizado em três áreas urbanas brasileiras (Almeida-Filho et al., 1992) mostrou que as pessoas solteiras apresentavam menor ocorrência de transtornos mentais não-psicóticos, mesmo controlando-se o efeito de outras variáveis, como sexo, idade e escolaridade (Coutinho et al., 1993).

Embora alguns trabalhos demonstrem a relação entre níveis mais altos de renda e consumo de psicofármacos (o inverso do que foi aqui observado), estes achados não são unânimes na literatura (Zadoroznys \& Svarstad, 1990; Dunbar et al., 1989). Mesmo no nosso trabalho, ao controlarmos a renda em relação às demais variáveis demográficas, a diferença entre os estratos sociais se enfraquece um pouco, embora o valor de $\mathrm{p}$ seja menor que 0,10 (Tabela 2).

Como em todos os estudos, os benzodiazepínicos permanecem na preferência absoluta dos usuários (e/ou de quem prescreve). Entre eles, destaca-se o diazepam (Woods et al., 1992). Embora os estudos sobre prescrição médica de benzodiazepínicos apontem os distúrbios psíquicos como a causa mais freqüiente de indicação, estudos com usuários não-psiquiátricos mostram que o seu uso está comumente ligado às desordens circulatórias, onde a ansiedade atua como um componente importante, em especial no caso da hipertensão arterial (Woods et al., 1992;
Stela, 1991). Um fato que nos chamou a atenção, entretanto, foi a ausência quase completa de antidepressivos, pois, atualmente, a prescrição deste tipo de psicofármaco já é uma prática entre os clínicos de diversas especialidades, o que não ocorre com os neurolépticos e anticonvulsivantes (Gentil-Filho, 1992). Além disso, a XX RA tem uma razoável rede pública de saúde, incluindo um hospital universitário (Hospital Universitário Clementino Fraga Filho), que dispõe de um serviço de psicologia médica e saúde mental, o qual, além da assistência direta, presta assessoria às demais especialidades. Há também na região uma unidade mista (Posto de Assistência Médica da Ilha do Governador, do Inamps) que oferece atendimento psiquiátrico ambulatorial. Isto nos conduz a algumas hipóteses. Em primeiro lugar, à questão do diagnóstico, uma vez que as síndromes depressivas leves, tão freqüentes em unidades primárias e secundárias de saúde, traduzindo-se em inúmeras queixas somáticas, podem estar sendo diagnosticadas como síndromes ansiosas, o que justificaria a prescrição do ansiolítico.

Caetano \& Stela (1990), em um estudo sobre prevalência de transtornos depressivos em um centro de atenção primária, verificaram que $39,8 \%$ dos pacientes apresentavam transtornos depressivos, sendo que apenas $17 \%$ dos pacientes foram diagnosticados pelo clínico geral.

O ansiolítico, por sua vez, é considerado um medicamento relativamente mais seguro e que oferece menos problemas (efeitos colaterais, risco de intoxicação, interações medicamentosas) do que os demais psicofármacos, inclusive o antidepressivo. Este fato pode fazer com que um clínico sinta-se seguro o suficiente para decidir incorporar o benzodiazepínico em seu arsenal terapêutico, mas não outros psicofármacos.

Por outro lado, Sonenreich (1988) chama a atenção para as divergências existentes em pesquisas com psicofármacos em matéria de indicações, dosagem e modo de ação, o que não contribui para dar aos especialistas e não-especialistas uma base sólida que conduza a uma prescrição adequada de tais medicamentos. Coutinho et al. (1988), em seu trabalho sobre a confiabilidade do diagnóstico em hospitais psiquiátricos da cidade do Rio de Janeiro, mostraram a baixa concordância diagnóstica existente 
nas avaliações registradas em prontuários médicos para um mesmo paciente.

Uma outra hipótese pode ter relação com o usuário, que, conforme observado em nossa prática clínica, classifica um benzodiazepínico como "um calmante mais fraco" e um antidepressivo como "um calmante muito forte". Além disso, como já foi referido, os efeitos colaterais dos antidepressivos existentes até então no mercado, em comparação com os efeitos colaterais dos benzodiazepínicos, podem contribuir para que o usuário reforce a idéia da relação "forte/fraco", fazendo com que ele não os utilize. Outro fator para a ausência de antidepressivos, embora um pouco menos provável, é o aumento do custo destas medicações, sobretudo os antidepressivos chamados de nova geração, tornando-os inacessíveis para uma boa parcela da população (Súmula, 1991). Evidentemente, qualquer uma dessas hipóteses nos remete a novos estudos, de natureza qualitativa, junto àqueles que prescrevem e àqueles que utilizam psicofármacos. Um exemplo deste tipo de estudo foi realizado por Armstrong et al. (1992), enfatizando que os generalistas acreditam que os problemas psicológicos inespecíficos são menos freqüentes do que crêem os psiquiatras.

A questão do diagnóstico dos distúrbios psíquicos e do manejo adequado dos psicofármacos torna-se mais relevante quando constatamos, tal como fizeram outros autores (Mari et al., 1992; Tancredi, 1979), que é o clínico geral quem está prescrevendo com maior frequiência os psicofármacos. Gentil-Filho (1992) coloca sua preocupação para com a formação oferecida pela maioria dos cursos médicos, onde os conhecimentos de psicofarmacologia ministrados no período da graduação não são suficientes para a tarefa de diagnosticar e tratar estes distúrbios. Porém, concordamos com Sonenreich (1988), quando diz que, concomitantemente, é preciso aprimorar o arcabouço teórico que delimita e adequa os arsenais terapêuticos à clínica dos distúrbios psíquicos.

Outro ponto que nos chama a atenção nos dados obtidos é a fonte de aquisição - cerca de $80 \%$ na rede de farmácias e drogarias do comércio, quando a substância ativa mais consumida é o diazepam, que é distribuído pela rede pública. Além dele, a carbamazepina, o fenobarbital e a imipramina também fazem parte da lista de medicamentos essenciais da CEME/MS. O abastecimento irregular das unidades públicas de saúde, com interrupções freqüientes de estoque das medicações, pode ser o responsável por esta distorção (Lucchesi, 1991; Medici et al., 1991).

Finalmente, gostaríamos de ressaltar que os estudos quantitativos são fontes importantes de hipóteses que conduzem a novas investigações, num processo de conhecimento gradual da realidade com a qual trabalhamos, funcionando também como uma avaliação da nossa prática. Com base nesta idéia, acreditamos ser importante desenvolver estudos, no nosso meio, sobre medicamentos que possam, conforme sugerem Van Der Geest \& Hardon (1988), ter enfoque nas pessoas que lidam com os medicamentos (provedores e consumidores) e nos temas (patologias e fármacos) a eles referentes.

Para os profissionais de saúde que já atuam na rede de serviços, seria útil a organização de um programa de educação continuada. A difusão de informações científicas adquire um importante papel na atualização do conhecimento, numa área em que muitas mudanças, tanto nos critérios diagnósticos quanto no arsenal terapêutico, têm ocorrido em um curto intervalo de tempo.

\section{RESUMO}

ALMEIDA, L. M.; COUTINHO, E. S. F. \& PEPE, V. L. E. Consumo de Psicofármacos em uma Região Administrativa do Rio de Janeiro: A Ilha do Governador. Cad. Saúde Públ., Rio de Janeiro, 10(1): 05-16, jan/mar, 1994.

Em 1988, um inquérito epidemiológico foi realizado para se estimar a prevalência de alcoolismo crônico e uso de álcool na população da XX Região Administrativa da cidade do Rio de Janeiro. O instrumento incluiu perguntas sobre o consumo de psicofármacos, café e cigarros. Este trabalho analisa os dados referentes ao consumo de psicofármacos nos 30 dias anteriores à pesquisa. A amostra representativa da população acima de 13 anos foi composta por 
1.459 pessoas, Os resultados mostram uma prevalência de consumo global de 5,2\% (3,1\% para homens e $6,7 \%$ para mulheres). As mulheres, as pessoas mais velhas (especialmente pessoas entre 60 e 69 anos), os separados e viúvos, e as pessoas de renda mais baixa apresentaram prevalências mais elevadas. Avaliou-se a associação de cada variável demográfica através do cálculo de odds ratio ajustado por regressão logística. Os tranqüilizantes derivados dos benzodiazepínicos predominaram entre os tipos de psicofármacos referidos $(85,23 \%)$, seguidos pelos antiepilépticos $(5,68 \%)$ e pelos hipnóticos e sedativos $(4,54 \%)$. Os clínicos não-especializados em neurologia ou psiquiatria lideraram as prescrições $(65,8 \%)$. Oitenta por cento dos medicamentos foram obtidos na rede privada, enquanto $13,16 \%$ foram adquiridos na farmácia pública, quase todos mediante a apresentação do receituário controlado. Os resultados são discutidos e novas linhas de pesquisa são apontadas. Palavras-Chave: Psicofármacos; Ansiolíticos; Epidemiologia; Prevalência; Consumo

\section{REFERÊNCIAS BIBLIOGRÁFICAS}

ALMEIDA, L. M. \& COUTINHO, E. S. F., 1993. Prevalência de alcoolismo crônico e de uso de álcool em uma região metropolitana do Rio de Janeiro, Brasil. Revista de Saúde Pública, 27: 23-29.

ALMEIDA-FILHO, N; MARI, J. J.; COUTINHO, E. S. F.; FRANÇA, J. F.; FERNANDES, J. G.; ANDREOLI, S. B. \& BUSNELLO, E. A., 1992. Estudo multicêntrico de morbidade psquiátrica em áreas urbanas brasileiras. Revista da Associação Brasileira de Psiquiatria - Associação Psiquiátrica da América Latina, 14: 93-104.

APPELBAUM, P.S., 1992. Controlling prescription of benzodiazepines. Hospital and Community Psychiatry, 43: 17-23.

ARMSTRONG, D. et al., 1992. Perceptions of psychological problems in general practice: a comparision of general practitioners and psychiatrics. Family Practice, 9: 173-176.

BERMUDEZ, J., 1992. Remédios: Saúde ou Indústria? A Produção de Medicamentos no Brasil. Rio de Janeiro: Relume-Dumará.
BOLTANSKI, L.; 1989. As Classes Sociais e o Corpo. $3^{\mathrm{a}}$ ed., Rio de Janeiro: Graal.

CAFFERATA, G. L. \& MEYERS, S. M., 1990. Pathways to psychotropic drugs - Understanding the basic of gender differences. Medical Care, 28: 285-298.

CAETANO, D. \& STELA, F., 1990. Prevalência de depressão em um centro de atenção primária. Fornal Brasileiro de Psiquiatria, 39 (supl. 1): 05-08.

CEME/MS (Central de Medicamentos do Ministério da Saúde), 1987. Medicamentos Essenciais: Os caminhos da autonomia. Documento-Proposta. Brasília, DF: CEME/MS. (Mimeo.)

COUTINHO, E. S. F; ALMEIDA-FILHO, N. \& MARI, J. J., s/d. Gênero, Situação Conjugal e Transtornos Psiquiátricos Menores. (Mimeo.)

COUTINHO, E. S. F.; KLEIN, C. H.; MORGADO, A. F.; IGUCHI, T. \& SOUZA, E. R., 1988. Confiabilidade do diagnóstico psiquiátrico em hospitais do Rio de Janeiro. Fornal Brasileiro de Psiquiatria, 37: 197-200.

DUNBAR, G. C.; PEREIRA, M. H. \& JENNER, F. A., 1989. Patterns of benzodiazepine use in Great Britain as measured by a general population survey. British Fournal of Psychiatry, 155: 836-841.

FIBGE (Fundação Instituto Brasileiro de Geografia e Estatística), 1983. Censo Demográfico: Dados gerais, migração, instrução, fecundidade, mortalidade. Rio de Janeiro: IBGE.

GENTIL-FILHO, V., 1992. Psicofarmacologia - 40 anos: Uma síntese crítica. Revista de Psiquiatria Clínica, 15-19 (especial): 17-23.

GUEREJE, O. \& OBIKOYA, B., 1991. Psychotropic drug use in an urban primary care clinic. Social Psychiatry Psychiatric Epidemiology, 26: 143146.

HOSMER JR, D.W. \& LEMESHOW, S., 1989. Appelied Logistic Regression. New York: John Wiley \& Sons.

KAHN, H. A. \& SEMPOS, C. T., 1989. Statistical Methods in Epidemiology. New York: Oxford University Press.

LAPORTE, J. R.; CAPELLA, D.; PORTA, M. \& FRATI, M. E., 1983. Patterns of use of psychotropic drugs in Spain in an international perspective. In: Clinical Pharmacology in Psychiatry. Brigding the experimental-therapeutic gap (L. F. Gram, org.), pp. 18-31, London: Macmillan.

LUCCHESI, G., 1991. Dependência e Autonomia do Setor Farmacêutico - Um Estudo da CEME. Tese de Mestrado, Rio de Janeiro: Escola Nacional de Saúde Pública, Fundação Oswaldo Cruz. 
MADUREIRA, P. R.; DE CAPITANI, E. M. \& CAMPOS, G. W. S., 1989. Avaliação da qualidade da atenção à saúde na rede básica. Cadernos de Saúde Pública, 5: 45-49.

MARI, J. J.; ALMEIDA-FILHO, N.; COUTINHO, E. S. F.; ANDREOLI, S. B.; MIRANDA, C. T. \& STREINER, D., s/d. The Epidemiology of Psychotropic Use in the city of São Paulo. Psychological Medicine. (no prelo)

MASUR, J. \& MOTEIRO, M. G., 1983. Validation of the "CAGE" alcoholism screening test in a Brazilian psychiatry inpatient hospital setting. Fournal of Biological Research, 16: 215-218.

MEDICI, A. C.; OLIVEIRA, F. \& BELTRÃO, K. I., 1991. A Política de Medicamentos no Brasil. Relatório técnico do IBGE. $\mathrm{N}^{\mathrm{o}}$ 1, Rio de Janeiro: IBGE.

MELLINGER, G. D.; BALTER, M. B. \& UHLENHUTH, E. H., 1984. Prevalence and correlates of the long-term regular use of anxiolytics. Fournal of the American Medical Association, 251: 376-379.

, 1985. Insomnia and its treatment prevalence and correlates. Archives of General Psychiatry, 42: 225-232.

NORTH, D. A.; McAVOY, B. R. \& POWELL, A. M., 1992. Benzodiazepine use in general practice - it is a problem? New Zealand Medical Fournal, 105:287-289.

OMS (Organization Mondiale de la Santé), 1990. $L a$ Situation Pharmaceutique dans le Monde. Genève: OMS.

PINDER, R. M., 1992. Os benefícios e os riscos das drogas antidepressivas. Fornal Brasileiro de Psiquiatria, 41 (supl. 1): 45-55.

ROCHA, F. L., 1992. A psiquiatria biológica em pauta. Fornal Brasileiro de Psiquiatria, 41: 8790.

ROZENFELD, S., 1989. O uso dos medicamentos no Brasil. In: Epidemiologia do Medicamento Princípios Gerais (J. R. Laporte; G. Tognoni \& S. Rozenfeld, orgs.), pp. 21-41, São Paulo: Hucitec/Rio de Janeiro: Abrasco.

SHADER, R. I.; GREENBLATT, D. J. \& BALTER, M. B., 1991. Appropriate use and regulatory control of benzodiazepines. Fournal of Clinical Pharmacology, 31: 781-784.
SONENREICH, C. 1988. Reflexões do clínico sobre farmacoterapia. Fornal Brasileiro de Psiquiatria, 37: 137-139.

STELA, F., 1991. Avaliação de traços e estados de ansiedade em pacientes hipertensos de centro de saúde. Fornal Brasileiro de Psiquiatria, 40: 497499.

SÚMULA, 1991. Indústria farmacêutica - Sem remédio para a fome do lucro. $\mathrm{N}^{\circ} 40$, página 1 , Ano VIII, Março. Rio de Janeiro, Projeto Radis/ Fiocruz.

TANCREDI, F. B., 1979. Aspectos Epidemiológicos do Consumo de Medicamentos Psicotrópicos pela População de Adultos do Distrito de São Paulo. Tese de Mestrado, São Paulo: Faculdade de Saúde Pública, Universidade de São Paulo. , 1986. Consumo de Medicamentos Benzodiazepínicos no Brasil - 1970-1985: Análise Comparativa de Tendências. Tese de Doutorado, São Paulo: Faculdade de Saúde Pública, Universidade de São Paulo.

VAN DER GEEST, S. \& HARDON, A., 1988. Drugs use: Methodological suggestions for field research in developing countries. Health Policy and Planning, 3: 152-158.

WHO/NORDISKA LAKEMEDELSNAMNDEN, 1993. Guidelines for ATC Classification. Norway: WHO Collaborating Centre for Drug Statistic Methodology/Sweden: Nordic Council on Medicines.

WOODS, J. H.; KATZ, J. L. \& WINGER, G., 1988. Use and abuse of benzodiazepines: Issues relevant to prescribing. Fournal of the American Medical Association, 260: 3476-5380. , 1992. Benzodiazepines: Use, abuse and consequences. Pharmacological Reviews, 44: $1-358$.

ZADOROZNYS, M. \& SVARSTAD, B. L., 1990. Gender, employment and medication use. Social Science E Medicine, 31: 971-978. 